\title{
Las industrias extractivas y sus implicaciones políticas y económicas ${ }^{+}$
}

\author{
FARID KAHHAT* \\ Pontificia Universidad Católica del Perú \\ fkahhat@pucp.edu.pe \\ https://doi.org/10.18800/rcpg.201601.007
}

\section{Resumen}

El artículo revisa la literatura académica sobre la relación entre dependencia de industrias extractivas de exportación y desempeño económico y político en Estados en desarrollo, elaborando un estado de la cuestión. Revisa primero la relación entre dependencia de industrias extractivas de exportación y violencia política, y discute por qué hay más guerras civiles en países que dependen de esas industrias. Revisa luego la relación entre dependencia de industrias extractivas de exportación y régimen político, y discute la influencia que esa dependencia puede tener sobre las perspectivas de democratización. Revisa finalmente la relación entre dependencia de industrias extractivas de exportación y desempeńo económico e institucional (es decir, la denominada "Maldición de los Recursos").

Palabras clave: Industrias extractivas, guerra civil, régimen político, desempeño económico, desarrollo institucional.

\section{The extractive industries and their political and economic implications.}

\begin{abstract}
This article reviews the academic literature on the relationship between dependency on extractive industries for export and economic and political performance in developing countries, presenting a state of the art. It first reviews the relationship between dependency on extractive industries for export and political violence, discussing why there are more civil wars in countries that show that dependence. It then reviews the relationship between dependency on extractive industries for export and political regime, discussing the influence that such a dependency might have over the prospects for democracy. It finally reviews the relationship between dependency on extractive industries for export and economic and institutional performance, i.e., the so called "Resource Curse".

Key words: Extractive industries, civil war, political regime, economic performance, institutional development.

\footnotetext{
* Docente del Departamento Académico de Ciencias Sociales. Coordinador de la Especialidad de Ciencia Política y Gobierno de la Facultad de Ciencias Sociales de la PUCP.

+ El presente artículo es una versión publicada en la Revista Estudios Internacionales vol. 45 no. 174 (enero 2013) del Instituto de Estudios Internacionales de la Universidad de Chile.
} 



\section{INTRODUCCIÓN}

Durante la década de los noventa numerosas investigaciones empíricas y estudios de caso encontraron una elevada relación entre dependencia de industrias extractivas (minería e hidrocarburos) y desempeño económico y político en los Estados en desarrollo. Específicamente, esa literatura postulaba que a mayor dependencia de industrias extractivas, mayor era la probabilidad de que un Estado padeciera de un bajo crecimiento económico, una baja calidad institucional, un régimen autoritario y un conflicto armado.

Esos hallazgos fueron, sin embargo, sometidos a un escrutinio crítico durante la siguiente década. En general, los estudios más recientes formulan tres tipos de cuestionamientos a sus antecesores. En primer lugar, la evidencia empírica no siempre es concluyente respecto a la relación entre las variables estipuladas por estudios previos. En segundo lugar, incluso cuando se acepta la relación entre esas variables, se cuestiona la dirección de la causalidad o los indicadores elegidos para medirlas. Por último, se intenta dilucidar el grado de sensibilidad de los hallazgos a cambios en las especificaciones.

Un ejemplo del tipo de debates a que han dado lugar esos cuestionamientos es el concerniente a la relación entre dependencia de industrias extractivas y desempeño de la economía. Mientras Sachs y Warner (1997) encuentran una relación inversa entre los recursos primarios como proporción del total de exportaciones y el nivel de crecimiento económico de un Estado entre 1970 y 1990, Brunschweiler y Bulte (2008) encuentran una relación directa entre esas variables para el periodo comprendido entre 1970 y 2000. Pero además de la diferencia en el rango de tiempo analizado, ambos estudios se diferencian en el hecho de que Brunschweiler y Bulte no postulan como variable independiente la proporción que los recursos primarios representan dentro del total de las exportaciones, dado que eso mide el grado de dependencia de la economía respecto a la dotación de recursos, y no su abundancia relativa. Y en ese caso la relación inversa podría ser espuria, en tanto tal vez la dependencia de la exportación de recursos primarios no sea lo que explica el bajo nivel de crecimiento económico: podría ser que tanto los bajos niveles de crecimiento como la dependencia de la exportación primaria se expliquen por las políticas (económicas y de desarrollo) que aplica el Gobierno.

Al postular como variable independiente no la proporción que las exportaciones primarias representan dentro de las exportaciones totales o del 
PBI, sino la abundancia relativa de recursos primarios, ${ }^{1}$ la relación entre recursos primarios y crecimiento económico se invierte: a mayor abundancia relativa se da un mayor crecimiento económico entre los Estados de la muestra para el periodo comprendido entre 1970 y 2000 (una muestra que es menor a la de Sachs y Warner, dado que solo incluye Estados con una relativa abundancia de recursos primarios).

Dada la relevancia de los temas involucrados y la controversia que existe en torno a ellos, este documento se propone realizar una revisión crítica de la literatura académica sobre la relación entre dependencia de industrias extractivas y desempeño económico y político en Estados en desarrollo, intentando bosquejar el estado de la cuestión. Revisaremos en primer lugar aquella literatura que analiza la relación entre la dependencia de industrias extractivas de exportación y la violencia política, intentando explicar por qué las guerras civiles ocurren de manera desproporcionada en Estados cuya economía depende de esas industrias, y por qué en esos casos se incrementa la probabilidad de que estas tengan una agenda secesionista. Revisaremos en segundo lugar la literatura sobre la relación entre industrias extractivas de exportación y régimen político, parte de la cual sugiere que la dependencia de esas industrias reduce la probabilidad de que un Estado tenga un régimen democrático. Intentaremos luego entender por qué la presunta relación entre industrias extractivas, de un lado, y violencia y autoritarismo, de otro, admite una excepción notable: la región de América Latina. Revisaremos por último el cuerpo de literatura que asocia la dependencia de actividades extractivas de exportación con un pobre desempeño económico y un bajo grado de desarrollo institucional (lo que se da en llamar «la maldición de los recursos»). Veremos, sin embargo, que la investigación más reciente sugiere que esa dista de ser una asociación necesaria.

\section{Industrias extractivas y guerras civiles}

El modelo Collier-Hoeffler (Collier y Sambanis 2005) es un aporte al estudio de las guerras civiles basado en una regresión que incluye una proporción significativa de los casos comprendidos entre 1960 y 1999. Una de sus conclusiones es hoy un lugar común en la literatura académica sobre el tema

\footnotetext{
1 La variable «abundancia de recursos primarios» y los criterios para su medición son tomados por los autores de un estudio del Banco Mundial. Esa variable «captura el valor descontado de las rentas esperadas de los recursos para un periodo futuro de 20 a 25 ańos, calculado en dólares americanos per cápita para 1994. Contrario a la variable de recursos estándar (que mide flujos), estas variables de riqueza estiman stocks de recursos -tanto a nivel agregado como divididos por tipo de recurso, como minerales o activos en tierras de cultivo. Ofrecen por ende variables más intuitivas para medir la abundancia».
} 
de las guerras civiles. Según esta, las demandas y/o clivajes sociales y políticos no bastan para explicar el inicio de una guerra civil: de hecho, tienen una baja capacidad predictiva. Para explicar el inicio de una guerra civil suele ser más importante la existencia de condiciones propicias para la organización y el financiamiento de una insurgencia armada. A su vez, el que la economía de un país dependa en una alta proporción de recursos primarios de exportación es una de las condiciones que facilitan la financiación de una insurgencia armada.

Esto último concuerda con la evidencia disponible, dado que las guerras civiles ocurren fundamentalmente en Estados cuya economía depende en una alta proporción de la exportación de recursos primarios: mientras mayor es la proporción del PBI representado por las exportaciones primarias, mayor es el riesgo de que un Estado se vea involucrado en una guerra civil (Collier, Elliot, Hegre, Hoeffler, Reynal-Querol y Sambanis 2003), particularmente en el continente africano. ${ }^{2}$ El riesgo de guerra civil alcanza su nivel máximo cuando las exportaciones primarias representan entre un 25\% y un $32 \%$ del PBI (Collier y Sambanis 2005).

No es solo que el riesgo de guerra civil sea mayor en Estados cuya economía depende de la exportación de recursos primarios, sino que además aumenta la probabilidad de que esas guerras sean de carácter secesionista. En términos estadísticos, el riesgo adicional de una guerra secesionista en Estados en los que la dotación de esos recursos duplica el promedio internacional es de 8.2\% (Collier y Hoeffler 2002).

Sin embargo, algunos estudios de caso sugieren que, al menos en los Estados involucrados, la correlación entre dependencia de recursos primarios de exportación y guerra civil podría ser espuria: en algunos casos en los que confluyen ambas variables, esos recursos no habrían sido ni el motivo ni la fuente de financiamiento de esas guerras (por ejemplo, en Nigeria, Mali, Senegal o Azerbaiyán) (Zinn 2005, Humpreys y Mohamed 2005, Zurcher, Baev y Koehler 2005). Pero incluso en los casos en los que esa confluencia podría estar asociada a una relación causal, existen problemas con la variable identificada por el modelo Collier-Hoeffler (exportaciones de recursos primarios como proporción del $\mathrm{PBI}$ ): esta incluye las exportaciones agrícolas, actividad en la que (salvo en el caso de la producción para el narcotráfico) no es fácil apropiarse de la producción o extraer rentas de ella sin tener antes un amplio control territorial.

2 Ver, por ejemplo, Ross (2004), Collier y Hoeffler (2005), y Fearon (2005). 
Entre los recursos primarios de exportación, la dependencia de recursos extractivos parece tener un mayor poder explicativo tanto del riesgo de que se inicie una guerra civil en un determinado Estado, como de la probabilidad de que se trate de una guerra secesionista. Por ejemplo, la probabilidad de que una guerra civil tenga una agenda secesionista es de 67.6\% en Estados que carecen de petróleo, pero se eleva a un $99.5 \%$ en Estados que poseen ese recurso (Collier y Hoeffler 2002). La explicación se basa en una característica medular que suelen compartir los recursos extractivos: su concentración en un área geográfica particular. Suelen además compartir dos características adicionales que también contribuyen a explicar la relación: de un lado, en su proceso de explotación los inversionistas privados suelen incurrir en costos hundidos sumamente elevados. De otro lado, son actividades que pueden generar rentas sumamente elevadas (es decir, retornos sobre la inversión mayores al promedio) (Sinnot, Nash y De La Torre 2010).

La concentración geográfica de esos recursos implica que un grupo insurgente podría apropiarse de ellos sin necesidad de lograr un amplio control territorial. El que los costos hundidos sean elevados, podría implicar que los inversionistas privados continúen con la producción incluso en presencia de una conducta predatoria por parte de la entidad política que controla el área geográfica en la que operan (Collier 2000 y Ross 2004). Incluso en aquellos casos en que los inversionistas privados no incurren en costos hundidos particularmente elevados (por ejemplo, la extracción de diamantes), podrían tener incentivos para continuar la producción en presencia de una conducta predatoria si los niveles de renta generados por la actividad extractiva son relativamente altos (Collier y otros 2003). Por último, si el área geográfica en la que se concentra el recurso es habitada mayoritariamente por miembros de un grupo étnico que no ejerce mayor control sobre las rentas que este genera, se eleva la probabilidad de que una eventual guerra civil tenga un carácter secesionista (Sudán del sur). Es decir, si las características de los recursos extractivos antes descritas coinciden con una desigualdad horizontal (desigualdad socio-económica entre grupos étnicos), la probabilidad de que una guerra civil tenga un carácter secesionista tiende a crecer (Ross 2004). ${ }^{3}$

Esta literatura no explica sin embargo por qué en varios países de América Latina una elevada dependencia de recursos extractivos no está asociada ni al

\footnotetext{
3 «Un desarrollo reciente y especialmente sorprendente es que los grupos rebeldes recaudan fondos vendiendo los derechos anticipados de la extracción de minerales que actualmente no controlan, pero los cuales se proponen controlar comprando armamentos financiados por medio de la venta de esos derechos de extracción» (Collier y otros 2003; 67).
} 
inicio de guerras civiles, ni a la existencia de conflictos secesionistas. Pese a existir un gran número de conflictos sociales relacionados con las industrias extractivas, estos no han dado lugar a insurgencias armadas, ni se ha producido una guerra secesionista en la región desde el siglo XIX.

Dos circunstancias históricas propias de la región contribuyen a explicar esa anomalía (Sinnot, Nash y De La Torre 2010). La primera es que, mientras la mayoría de los Estados en Asia y África se crean recién a partir del final de la Segunda Guerra Mundial, la virtual totalidad de los Estados latinoamericanos existen como entidades soberanas desde el primer tercio del siglo XIX. No solo no ha surgido un nuevo Estado en la región en más de un siglo, sino que la mayoría de los límites entre Estados se definieron entre el siglo XIX y la primera mitad del siglo XX. Y en la mayoría de casos en que los diferendos limítrofes se prolongaron en el tiempo, estos han sido abordados a través de una negociación bilateral (Argentina y Chile), un arbitraje (Ecuador y Perú) o la competencia contenciosa de la Corte Internacional de Justicia (Honduras y Nicaragua).

La segunda razón es que, hasta hace relativamente poco tiempo, las desigualdades horizontales de la región no habían propiciado la politización de las identidades étnicas. Y cuando esa politización finalmente se produjo, esta no involucró la adopción de una agenda secesionista. Por ejemplo, la Confederación de Nacionalidades Indígenas del Ecuador (CONAIE) promovió una reforma constitucional según la cual la pertenencia a la nación se definía por un criterio cívico: se definía como ecuatoriano a todo aquel que poseía la ciudadanía del país, y todos los ciudadanos debían ser iguales en derechos y deberes ante la ley, con prescindencia de su cultura (lo cual podría ser suscrito por cualquier liberal). Pero para la CONAIE la diversidad cultural es un bien público que las políticas del Estado deberían promover, y el reconocimiento de derechos sociales sería una condición para que la igualdad de oportunidades no sea una mera formalidad legal (cosa que algunos liberales tendrían problemas en suscribir). La única excepción a esa regla es la del Movimiento Indígena Pachakuti en Bolivia (que promueve la creación de lo que denomina la «República Indígena del Collasuyo»), pero se trata de una fuerza política marginal en el espectro político boliviano (como demuestran sus resultados electorales).

Estudios recientes podrían contribuir a explicar desde otra perspectiva esta excepcionalidad latinoamericana. Admitiendo que existe una relación entre la dependencia de industrias extractivas y la probabilidad de que se inicie una guerra civil en un Estado, esos estudios invierten la dirección causal: la guerra civil sería la variable que explica la dependencia de la economía de las industrias 
extractivas. Las características antes descritas (concentración geográfica, costos hundidos y generación de rentas) y sus escasos eslabonamientos con la economía nacional (demandan pocos insumos de ella y exportan la mayor parte de su producción) explicarían esa relación: ${ }^{4}$ Las industrias extractivas podrían continuar operando en condiciones en las que una guerra civil provocaría el colapso de actividades económicas con mayor movilidad, menores rentas o mayores eslabonamientos con la economía nacional (Brunschweiler 2008). Esa información parece consistente con los hallazgos de un reporte reciente del Banco Mundial, según el cual la violencia política se está convirtiendo en la principal causa de la pobreza en un número creciente de Estados (Banco Mundial 2011). Ello se debe a que se trata de Estados involucrados en ciclos recurrentes de violencia política: el 90\% de los 39 Estados que sufrieron guerras civiles desde el año 2000, habían padecido ya una guerra civil durante las tres décadas previas. 5 En esos casos, el comercio de drogas y las exportaciones de las industrias extractivas han sido fuentes privilegiadas de financiamiento de la violencia política, sobre todo después de que esa violencia produjera el virtual colapso del resto de la actividad económica.

La crítica no solo consiste en invertir la dirección causal (son las guerras civiles las que causan la dependencia de industrias extractivas y no al revés), sino además en sostener que la abundancia de recursos primarios reduce de manera indirecta la probabilidad de que se inicie una guerra civil, al incrementar el nivel de ingresos (las guerras civiles se producen de manera desproporcionada en Estados con un bajo nivel de ingreso per cápita): un incremento de una desviación estándar en la abundancia relativa de recursos primarios reduciría en algo menos de un 5\% la probabilidad de guerra civil (Brunschweiler 2008). Esos estudios no niegan la posibilidad de que en casos específicos las industrias extractivas estén asociadas al inicio de guerras civiles, pero sí cuestionan la premisa de que se trata de un patrón general.

\footnotetext{
4 «En razón de las elevadas rentas que normalmente se involucran en su producción y su relativa independencia de insumos del resto de la economía, las exportaciones de recursos naturales son relativamente sólidas frente al conflicto. En comparación, exportaciones más complejas son comúnmente de bajo margen y dependen de una frágil red de interdependencias comerciales, y estas últimas muestran la tendencia a verse gravemente perturbadas por la guerra» (Collier y otros 2003: 74).

5 «Muchos países y zonas subnacionales atraviesan ahora ciclos de violencia repetida, [...] los conflictos muchas veces no son episodios aislados sino que se prolongan en el tiempo y se repiten: el $90 \%$ de las guerras civiles del último decenio tuvo lugar en países que habían sufrido ya una guerra civil en los últimos 30 años» (Collier y otros 2003: 2).
} 


\section{Industrias extractivas y régimen político}

Diversos estudios en varias regiones del mundo en desarrollo sugieren que mientras mayor sea la dependencia de la economía de un Estado de la exportación de recursos extractivos, menor será la probabilidad de que este tenga un régimen democrático. ${ }^{6}$ Esa relación es particularmente alta cuando se trata de un recurso extractivo de exportación en particular: el petróleo. Por ejemplo, entre los veinte principales exportadores de petróleo en el año 2000, solo México y Venezuela eran democracias representativas, e incluso esos países experimentaron periodos prolongados bajo égida autoritaria (de hecho, algunos autores califican al actual régimen venezolano como un «autoritarismo competitivo») (Karl 1997).

Existen cuatro mecanismos que podrían explicar la relación entre industrias extractivas y autoritarismo político (tres de los cuales derivan de las rentas que estas generan) (Ross 2010). El primero es que las rentas generadas por las industrias extractivas permiten incrementar la capacidad represiva del Estado. ${ }^{7}$ El segundo es que esas rentas pueden dotar al Estado de recursos para formar redes clientelares que le provean una base social. Sin embargo, ese argumento también sería válido bajo un régimen democrático. Por ejemplo, si parte de esas rentas se destinan a implementar políticas sociales, podría disminuir el respaldo electoral de aquellas fuerzas que promueven una redistribución del ingreso por vía autoritaria, lo cual a su vez haría a los inversionistas privados más proclives a aceptar la democracia. Es decir, antes que propiciar un determinado tipo de régimen político, este mecanismo propiciaría la estabilidad bajo el régimen político que existía al momento de generarse esas rentas.

Bahréin, en 2011, provee un ejemplo de la aplicación sucesiva de ambos mecanismos en una economía dotada de rentas producto de la exportación de petróleo: temiendo el contagio de las protestas que se suscitaban en el mundo árabe, la monarquía decide «obsequiar» a cada familia del reino el equivalente a unos 2,700 dólares como medida preventiva. Cuando esa medida no consigue impedir las protestas, la monarquía apela a la represión, utilizando para ese fin medios de un alto costo y sofisticación tecnológica, concebidos para una guerra

\footnotetext{
6 «Diversos estudios que exploraron esta relación encuentran que la riqueza mineral no sólo reduce la probabilidad de que se produzca una transición democrática, sino que además hace menos probable la consolidación de la democracia y, a su vez, promueve la consolidación de regímenes autoritarios» (Weinthal y Jones Luong 2006: 36).

7 En su reporte de 2010 sobre el estado de la democracia en el mundo, la unidad de inteligencia (EIU por sus siglas en inglés) de la revista The Economist comienza afirmando que «La democracia está ahora en repliegue. El patrón dominante en todas las regiones en los dos últimos ańos ha sido el retroceso respecto al progreso previo en materia de democratización» (EIU 2010: 1). Una de las razones que explicarían esa tendencia es que «Los autócratas han aprendido a protegerse mejor; muchos de ellos presiden Estados con grandes reservas de energía, y se han fortalecido por el incremento sostenido en los precios del petróleo» (EIU 2010: 2).
} 
convencional (tanques adquiridos de los Estados Unidos). Cuando la represión tampoco consiguió impedir las protestas, el régimen encaró la disyuntiva de escalar el nivel de la represión o iniciar negociaciones con la oposición, decantándose en lo esencial por la primera opción (a través de la intervención de tropas del Consejo de Cooperación del Golfo).

El tercer mecanismo que explicaría la relación entre industrias extractivas y autoritarismo político supone que las rentas que aquellas generan le permiten al Estado tener una fuente de ingresos que no depende de la existencia de una amplia base tributaria. Al no depender los ingresos fiscales de los tributos que paga la mayoría de los ciudadanos, estos tienen una menor capacidad para exigir al Estado una rendición de cuentas sobre la gestión de los recursos públicos (algo que, en principio, es una característica propia de los regímenes democráticos).

Ese mecanismo se basa en la presunción de que las rentas generadas por las industrias extractivas impiden el surgimiento de lo que algunos autores denominan un "contrato fiscal», que habría propiciado la liberalización de los sistemas políticos en Europa Occidental. La idea básica es que los avances en las tecnologías bélicas hicieron que las guerras interestatales tuvieran un costo creciente. Dado que la mayor parte de la riqueza era generada por empresas de propiedad privada, acceder a los recursos necesarios para financiar esas guerras requería de una mayor presión tributaria sobre esas empresas. Lo cual dotaba a estas últimas del poder de negociación necesario para exigir a cambio la garantía de ciertos derechos, comenzando por el derecho a la propiedad y al cumplimiento de los contratos. Es decir, los gobernantes aceptan establecer restricciones institucionales a su ejercicio del poder político, a cambio del acceso a nuevos recursos (Mahon 2003).

En Estados que poseen abundantes rentas producto de las industrias extractivas, los gobernantes podrían en principio obtener todos los recursos que requieran de los impuestos que pagan las empresas del sector, o de la explotación directa de los recursos extractivos (reduciendo la necesidad de obtener tributos del resto de la sociedad). Ello hace que no requieran negociar con los gobernados la concesión de derechos a cambio de la obtención de recursos (Levi 1988).

El cuarto mecanismo que contribuye a explicar la relación entre industrias extractivas y autoritarismo político se basa en el hecho de que esas industrias suelen generar economías de enclave, sin mayores eslabonamientos con el resto de la economía. Lo cual explica que no tiendan a producir el tipo de modernización social que propicia la democracia (por ejemplo, una 
diversificación ocupacional, con una clase media profesional relativamente grande como proporción de la población).

En general, la dependencia de industrias extractivas haría menos probable el surgimiento de grupos sociales que pudieran eventualmente demandar derechos civiles y políticos (trabajadores fabriles, clases medias profesionales, empresarios industriales), porque el Estado tendría menos incentivos para promover el desarrollo de industrias y servicios urbanos (que generan una demanda por mano de obra calificada) como fuente potencial de ingresos tributarios (Isham, Woolcock, Pritchett \& Busby 2002). Por ejemplo, una veta de la literatura sobre los orígenes sociales de la democracia concede gran importancia a una eventual alianza entre clases medias profesionales y trabajadores sindicalizados como fuerza motriz del proceso de democratización. ${ }^{8}$

Pero incluso allí donde una economía basada en industrias extractivas genera el tipo de grupos sociales antes mencionados, estos pueden ver restringida su capacidad de plantear demandas políticas por dos razones: la primera es que podrían carecer de una fuente independiente de ingresos, y depender del Estado como principal fuente de empleo (por ejemplo, en 1975 el Estado kuwaití empleaba al $75 \%$ de la población económicamente activa) (Crystal 1989). La segunda es que, en casos como el del Golfo Pérsico, la mano de obra (calificada o no) proviene en una alta proporción de inmigrantes que carecen de ciudadanía y, por ende, de los derechos civiles y políticos asociados a ella (en Bahréin, por ejemplo, el $54 \%$ de la población está compuesta por extranjeros residentes).

Por último, diversas investigaciones empíricas sugieren que una intervención militar extranjera guarda una relación entre insignificante e inversa con la probabilidad de que un Estado desarrolle una democracia estable: ello es así incluso cuando el Estado que interviene militarmente posee un régimen democrático, y uno de los propósitos declarados de su intervención es el de promover la democracia. ${ }^{9}$ A su vez, existen razones para suponer que los Estados

\footnotetext{
8 Ver, por ejemplo, Rueschemeyer, Stephens y Stephens (1992).

9 Un artículo reciente resume así los principales hallazgos de esa literatura: «Un estudio de 2006 de Jeffrey Pickering y Mark Pecency encontró que la intervención militar por parte de Estados liberales (como Gran Bretaña, Francia y Estados Unidos) "rara vez ha desempeńado un papel en procesos de democratización desde 1945". De manera similar, George Downs y Bruce Bueno de Mesquita de la Universidad de Nueva York encuentran que las intervenciones de los Estados Unidos desde la Segunda Guerra Mundial condujeron a democracias estables en la siguiente década en menos de 3\% de los casos, y un estudio [...] de William Easterly encuentra que tanto las intervenciones de los Estados Unidos como las de la Unión Soviética durante la Guerra Fría generalmente condujeron a "declives significativos en la democracia”. [...]. Otra investigación de Dawnes (en coautoría con Jonathan Monten de LSE) encuentra que "Estados cuyos gobiernos son derrocados por una democracia no tienen probabilidades sensiblemente mayores de convertirse en democracias que Estados similares que no experimentan una intervención”» (Walt 2011).
} 
con reservas de petróleo en Medio Oriente son particularmente proclives a padecer una intervención militar: en primer lugar, porque el petróleo es todavía una fuente de energía sin sustitutos costo-eficientes (es decir, capaces de proveer el mismo rendimiento a un costo equivalente), y es de uso tanto civil como militar. En segundo lugar, una proporción inusualmente elevada de las reservas internacionales de ese recurso se encuentra en el Medio Oriente. ${ }^{10}$ De ser así, ese sería otro nexo probable (aunque indirecto) entre abundancia de recursos extractivos y régimen político para el caso de una región particular.

Nuevamente, América Latina constituye una excepción a la regla según la cual la dependencia de industrias extractivas está asociada al autoritarismo político (Dunning 2008, Ross 2010, Haber y Menaldo 2009). Ecuador y Venezuela, por ejemplo, desarrollaron instituciones democráticas durante periodos de altas rentas producto de la exportación de petróleo.

Existen algunas explicaciones posibles para la excepcionalidad latinoamericana (Sinnot, Nash y De La Torre 2010). Una primera sugiere que el grado de dependencia de los recursos primarios de exportación hace una diferencia: mientras más dependa de ellos el conjunto de la economía (y no solo los ingresos fiscales), mayor será la probabilidad de que el régimen político sea autoritario (Dunning 2009). Por ejemplo, un estudio que clasifica a los Estados según ese grado de dependencia (medida como la proporción que representan los recursos naturales sobre el total de las exportaciones), coloca en los dos primeros lugares a Omán y Kuwait, mientras que el primer Estado de América Latina que aparece en la lista es Venezuela, en el puesto veintiuno (Sachs y Wagner 1997).

Otra explicación de la excepcionalidad latinoamericana involucra la elevada desigualdad en la distribución del ingreso, característica de la región. Esa elevada desigualdad podría incrementar el respaldo de alternativas que promueven la redistribución del ingreso bajo un régimen autoritario, lo cual a su vez reduce la probabilidad de que los inversionistas privados respalden ese tipo de régimen.

En general, una elevada desigualdad en la distribución del ingreso suele estar asociada a un elevado respaldo hacia políticas redistributivas. Y en economías altamente dependientes de las rentas generadas por la exportación de recursos

\footnotetext{
La ayuda económica (un instrumento que suele acompañar la intervención militar de Estados democráticos) tampoco parece hacer mayor diferencia: «Evidencia substancial respalda la conclusión de que la ayuda tiene sólo un efecto menor en los esfuerzos por mejorar el crecimiento económico, los servicios de salud, la educación, el bienestar social, la libertad o la rendición de cuentas por parte del gobierno" (Bueno de Mesquita 2006).

10 Diez países, en lo esencial provenientes de esa región, concentran más del $80 \%$ de las reservas probadas. Los Estados integrantes de la OPEP (la mayoría de los cuales provienen del Medio Oriente) poseen el $73.5 \%$ de esas reservas (El País 2011: 8).
} 
primarios, estas constituyen la principal fuente de ingresos disponible para su redistribución. A su vez, el control estatal sobre esas rentas podría tener un mayor efecto democratizador que su control por parte de una élite privada (como, por ejemplo, en Bolivia antes de 1952), en tanto hace más probable la adopción de políticas redistributivas (Dunning 2008).

En este caso, nuevamente, la excepcionalidad latinoamericana podría explicarse con base en las contribuciones más recientes de la literatura académica. Como en casos anteriores, una de esas contribuciones sostiene que la dependencia de recursos primarios no es una variable exógena, y que con especificaciones diferentes la dependencia de recursos primarios no aparece asociada ni con la erosión del régimen democrático, ni con una menor probabilidad de completar la transición del autoritarismo a la democracia. Sugiere además que una baja capacidad institucional del Estado estaría a la base tanto de la erosión del régimen democrático como de una mayor dependencia de la economía de recursos primarios de exportación (Haber y Menaldo 2011).

\section{Industrias extractivas y desempeño económico e institucional}

Diversos estudios sugieren que mientras mayor sea la dependencia de una economía de la exportación de recursos extractivos (medida como proporción del PBI), menor tenderá a ser su tasa de crecimiento (Auty y Gelb 2001, Sachs y Warner 2001, Sala-I-Martin y Subramain 2003 y Ross 2003). Por ejemplo, entre 1970 y 1993 el crecimiento promedio de Estados con una alta dependencia de exportaciones mineras o de hidrocarburos fue significativamente menor que el de Estados que carecían de esa dotación de recursos (Auty 1993).

Una razón que explicaría esa relación entre dependencia de exportaciones extractivas y desempeño económico es la denominada «enfermedad holandesa»: las elevadas rentas producidas durante periodos de bonanza exportadora producen:

[...] una apreciación del tipo de cambio (es decir, de la tasa de cambio entre monedas ajustada por la inflación) al trasladarse insumos productivos (capital y trabajo) hacia el sector extractivo y hacia el sector no transable (es decir, el comercio minorista, los servicios y la construcción), reduciendo por ende la competitividad de otros sectores de exportación (por ejemplo, agricultura e industria), precipitando así su colapso (Weinthal y Jones Luong 2006: 37).

Eso crea incentivos perversos, en tanto hacen menos probable la diversificación de la oferta exportable (Sinnot, Nash y De La Torre 2010), lo cual explica la vulnerabilidad de esas economías ante la marcada volatilidad en la cotización internacional de los recursos extractivos. Ello es particularmente 
cierto respecto a los precios de los minerales desde la Segunda Guerra Mundial: estos, por ejemplo, tuvieron una marcada tendencia declinante entre 1974 y 2003, para experimentar desde entonces un crecimiento sin precedentes.

La volatilidad en los precios internacionales de los recursos extractivos se explica por el hecho de que tanto la oferta como la demanda de esos bienes son relativamente inelásticas. A su vez, el hecho de que el desempeño del conjunto de la economía dependa de precios volátiles tiende a desincentivar la inversión privada, en tanto eleva los niveles de riesgo e incertidumbre que esta debe afrontar. Todo lo anterior, además, hace que los ingresos fiscales sean relativamente inestables.

Diversos estudios sostienen que los Estados que basan sus ingresos en rentas provenientes de la exportación de recursos extractivos suelen padecer deficiencias institucionales. ${ }^{11}$ Ese hallazgo es consistente con lo que sugieren otras fuentes. De un lado, esos Estados suelen ubicarse en posiciones rezagadas tanto en el Governance Research Indicators del Banco Mundial, ${ }^{12}$ como en el Corruption Perception Index de Transparencia Internacional. ${ }^{13}$ De otro lado, según el «Índice de Estados Fallidos» para 2006 de la revista Foreign Policy, el $72.2 \%$ de las reservas mundiales de petróleo y el $65.9 \%$ de las reservas mundiales de gas se ubicaban en países con algún riesgo de convertirse en Estados fallidos. ${ }^{14}$

La razón de esa relación sería que la conveniencia de reformas institucionales deriva en lo esencial de la necesidad de mejorar la eficiencia del Estado tanto en la recaudación de recursos como en su asignación. Pero si el Gobierno puede obtener ingresos elevados de las rentas extractivas, no tendrá los mismos incentivos para adoptar esas reformas institucionales. Eso, como se indicó con anterioridad, incluye la falta de incentivos para establecer un régimen fiscal moderno con una amplia base tributaria.

Pero, aunque común, la relación inversa entre dependencia de exportaciones extractivas, de un lado, y desempeño económico y eficiencia institucional, de otro, dista de ser una relación necesaria. La razón es simple: si bien los Estados suelen tener un control limitado sobre los precios internacionales de sus recursos extractivos, pueden adoptar medidas que amortigüen los impactos negativos de

\footnotetext{
11 Ver, por ejemplo, Mann (1988).

12 www.worldbank.org/wbi/governance/dada.html

13 www.transparency.org/surveys/index.html\#cpi.

14 Esos totales derivan de sumar las reservas ubicadas en Estados que, de acuerdo al índice, se encontraban dentro de cada una de las siguientes categorías: «Estados en el límite», «Estados en peligro» o «Estados en situación crítica» (ver http://www.fp-es.org/).
} 
la volatilidad en su cotización. De hecho, tal vez el tema de mayor interés en la literatura académica sea el intento de explicar por qué algunos Estados logran conjurar la denominada «maldición de los recursos», mientras otros sucumben ante ella. El contraste más evidente es el que existe entre experiencias como la de Noruega, de un lado, y las experiencias de varios Estados latinoamericanos, de otro (Blomstrom y Meller 1990). Una diferencia crucial entre esas experiencias deriva de la secuencia histórica de los acontecimientos: en el caso de Noruega, por ejemplo, la construcción de un Estado que gestione los asuntos públicos con transparencia, eficacia y rendición de cuentas precedió al descubrimiento y explotación de los recursos petrolíferos (Davis, Ossowski \& Fedelino 2003).

Pero incluso dentro de América Latina existen diferencias significativas en la administración de las rentas extractivas. En el área andina, por ejemplo, el Estado chileno representa un caso relativamente exitoso de administración de esas rentas, al menos por dos razones. La razón principal radica en un desarrollo relativamente temprano (fines del siglo XIX) de la capacidad institucional necesaria para administrar esos recursos de una manera que permitiera evitar la «enfermedad holandesa». Esa capacidad institucional se ve reflejada, por ejemplo, en el fondo de estabilización fiscal que le permite al Estado chileno desarrollar políticas contra cíclicas.

Una constante en la literatura es, por ejemplo, la constatación de las ventajas que reportan en materia de rentas extractivas la separación de poderes y la transparencia en su administración (Dunning 2008). La separación de poderes que crea una estructura de incentivos adecuada es aquella en la cual la autoridad para decidir qué proporción de esas rentas debe destinarse a un fondo de estabilización, es independiente de la autoridad para decidir cómo deben emplearse los recursos de ese fondo. Condición que cumple el Estado chileno. Porque dispone de un panel de expertos independientes del Gobierno de turno que decide sobre las tendencias de evolución en materia de rentas extractivas. En cuanto a la transparencia en la administración del fondo, en Chile existen normas que derivan de acuerdos políticos sobre la asignación de las rentas extractivas (es decir, el destino al que se asignan no depende de una decisión discrecional del Gobierno de turno). ${ }^{15}$

\footnotetext{
15 Durante décadas uno de los acuerdos políticos en torno al empleo de las rentas extractivas era la denominada «Ley Reservada del Cobre», según la cual el 10\% del total de las exportaciones de la empresa estatal exportadora de cobre (conocida por el acrónimo de Codelco) debían destinarse a la adquisición de armamento para las Fuerzas Armadas. Esa ley finalmente fue derogada en 2011.
} 
Por lo demás, ya desde 1939 el Estado había creado la Corporación para el Fomento de la Producción (CORFO), cuyo fin era promover tanto la diversificación como la descentralización de la economía.

Pero todo esto a su vez lleva nuevamente a preguntarse en qué medida la experiencia chilena es replicable, dado que el desarrollo institucional del Estado habría precedido cuando menos en parte a la bonanza en materia de rentas extractivas. El punto, sin embargo, sigue siendo que la capacidad de agencia de los actores políticos puede hacer una diferencia, suponiendo que puedan definir sus propios intereses dentro de un horizonte temporal de largo plazo. Eso explicaría por qué un proyecto de investigación reciente auspiciado por el Banco Mundial concluye que «el peso de la evidencia econométrica y los estudios de caso indican que la "maldición de los recursos" (la abundancia de recursos naturales reduce el crecimiento de largo plazo), de existir, no es ni fuerte ni inevitable» (Sinnot, Nash y De La Torre 2010: 57). Por lo demás, en lo que respecta a la relación entre desempeño económico y calidad institucional en Estados con una abundancia de recursos primarios de exportación, la evidencia empírica dista de ser concluyente. Existe, por ejemplo, un estudio que emplea la proporción que las exportaciones primarias representan dentro del ingreso nacional como indicador del grado de abundancia de recursos. Ese estudio concluye que mientras mayor sea el grado de abundancia, menores serán tanto la tasa de crecimiento económico como el grado de calidad institucional (Mehlum, Moene y Torvik 2006).

Pero esa conclusión no se sostiene cuando se cambian las especificaciones, en particular cuando se emplean criterios de medición del grado de abundancia que eviten los problemas de endogeneidad implícitos: de un lado, la posibilidad de que una alta proporción de exportaciones primarias dentro del ingreso nacional sea el resultado de bajas tasas de crecimiento, y no al revés (Sinnot, Nash y De La Torre 2010: 58). De otro, la posibilidad de que una alta proporción de exportaciones primarias dentro del ingreso nacional sea el resultado de una baja calidad institucional, dado que esta última tiende a reducir la rentabilidad de la inversión en otras actividades económicas (que, a diferencia de las exportaciones primarias, no contarían con altos niveles de renta).

Estudios que realizan cambios en las especificaciones o en los criterios de medición de variables como "abundancia de recursos primarios», concluyen que esta última no solo puede tener un efecto positivo sobre la tasa de crecimiento, sino además que ese efecto es mayor en Estados con una baja calidad institucional (Alexeev y Conrad 2009, Brunnschweiler 2008). Tanto 
porque las industrias extractivas suelen ser consideradas un sector «estratégico», ${ }^{16}$ como por las rentas y los costos hundidos que suelen involucrar, las empresas privadas del sector son particularmente vulnerables a intentos de expropiación. Eso es cierto sobre todo en el caso de las industrias de hidrocarburos. Pero la estructura de incentivos que enfrenta un Estado al momento de decidir si expropiar o no una determinada empresa incluye además otras variables. La literatura sugiere que las expropiaciones son más probables cuando el recurso involucrado tiene una alta cotización en los mercados internacionales, que cuando su cotización se mantiene a la baja. De hecho, con una cotización a la baja se incrementa la probabilidad de que los Estados privaticen empresas públicas del sector, presumiblemente para aprovechar la mayor eficiencia de la empresa privada (Sinnot, Nash y De La Torre 2010) (al margen del debate sobre la eficiencia relativa de las empresas públicas, el punto es que durante los periodos de bonanza estas no cuentan con mayores incentivos para mejorar la calidad y la transparencia de su gestión, particularmente bajo regímenes autoritarios).

Sin embargo, el efecto de la cotización internacional de un recurso extractivo sobre la probabilidad de expropiación depende en parte del régimen tributario y las condiciones contractuales bajo las cuales una determinada empresa opera: cuando estas contemplan cláusulas de contingencia relativas a la cotización internacional del recurso (como ocurre en el caso de Chile), el Estado puede beneficiarse de los periodos de bonanza sin necesidad de afectar los derechos de

\footnotetext{
16 Un criterio con base en el cual las autoridades políticas suelen calificar un determinado sector económico como «estratégico» es su incidencia sobre el desempeño de la economía en su conjunto. El otro suele ser su incidencia sobre la capacidad de defensa de un Estado, tanto en lo que se refiere a sus arsenales de armas como en lo que se refiere a su logística militar.
}

Un ejemplo del primer criterio son las razones que dio el Secretario de Tesoro, Henry Paulson, para explicar por qué el gobierno de los Estados Unidos decidió rescatar con fondos públicos en 2008 a dos empresas dedicadas a proveer crédito hipotecario: «Fannie Mae y Freddie Mac son tan grandes y están tan entrelazadas con nuestro sistema financiero que la quiebra de cualquiera de ellas causaría una gran turbulencia en los mercados financieros de nuestro país y alrededor del mundo. Esa turbulencia tendría un impacto negativo directo sobre la riqueza de los hogares: desde los presupuestos familiares hasta el valor de las casas, pasando por los ahorros para estudios y las pensiones de retiro. Una quiebra afectaría la capacidad de los estadounidenses de obtener créditos hipotecarios, de consumo o para financiar negocios. Y una quiebra tendría un efecto adverso sobre el crecimiento económico y la creación de puestos de trabajo. Por esos tomamos esta decisión el día de hoy» (DEALBOOK 2008).

Un ejemplo del segundo criterio para considerar un sector económico como "estratégico» (su incidencia sobre la capacidad de defensa de un Estado), es el de la creación del mecanismo de "Acciones Doradas» durante el gobierno de Margaret Thatcher en Gran Bretańa. Estas proveían al Estado británico de un derecho de veto sobre ciertas decisiones corporativas (esencialmente en temas relacionados con la propiedad, tales como la ampliación del capital, la adquisición o la fusión con otras empresas). El propósito solía ser evitar que empresas que producían tecnología de punta en el ámbito militar pudieran ser adquiridas por Estados considerados hostiles. 
propiedad (Sinnot, Nash y De La Torre 2010). Cuando ese no es el caso (como ocurre con los "convenios de estabilidad tributaria» suscritos por el Estado peruano, habitualmente durante periodos de baja cotización internacional), siempre es posible renegociar los convenios existentes de común acuerdo. Sin embargo, el fracaso de esa alternativa haría más probable una eventual expropiación.

Finalmente, un estudio señala que la probabilidad de expropiación estaría asociada además a otras tres variables. La primera es la dependencia de la economía del recurso extractivo involucrado (entendida como la proporción del PBI representada por las exportaciones): a mayor dependencia, mayor es la probabilidad de expropiación. La segunda variable es la calidad de las instituciones (medida con base en indicadores de institucionalidad democrática y de restricciones institucionales sobre el ejecutivo): a menor calidad institucional, mayor es la probabilidad de expropiación. La tercera variable es el nivel del capital humano de un Estado (medido con base en la tasa de alfabetización entre la población adulta): a menor nivel de capital humano, mayor probabilidad de expropiación (Guriev, Kolotilin \& Sonin 2008).

\section{Bibliografía}

Alexeev, M. y R. Conrad (2009). «The Elusive Curse of Oil». The Review of Economics and Statistics. 91 (3), pp. 586-598.

Auty, Richard (1993). Sustainable Development in Mineral Economies: The Resource Curse Thesis. London: New York: Routledge.

Auty, Richard y Alan GELB (2001). «Political Economy of Resource Abundant States». En Auty, Richard (Ed.). Resource Abundance and Economic Development. Oxford: Oxford University Press, pp. 126-144.

Banco Mundial (2011). Informe sobre el Desarrollo Mundial 2011: Conflicto, seguridad y desarrollo. Washington DC.: Banco Mundial. Consulta: 12 de octubre de 2016.

http://www.contexto.org/pdfs/BMdesarrllmundial2011Overview.pdf

Blomström, Magnus y Patricio Meller (Eds.) (1990). Trayectorias divergentes: Comparación de un siglo de desarrollo económico latinoamericano y escandinavo. Santiago de Chile: CIEPLAN.

Brunnschweiler, Christa (2008). «Cursing the Blessings? Natural Resource Abundance, Institutions and Economic Growth». World Development, 36 (3), pp. 399-419.

Brunschweiler, Christa y Erwin BULTE (2008). «Linking Natural Resources to Slow Growth and More Conflict». Science. Nro. 320, pp. 616-617.

Bueno de Mesquita, Bruce (2006). "Central Issues in the Study of International Conflict». En The Oxford Handbook of Political Economy. Oxford: Oxford University Press.

Collier, Paul (2000). Economic Causes of Civil Conflict and their Implications for Policy. World Bank.

Collier, Paul y Anke Hoeffler (2005). «Resource Rents, Governance, and Conflict». Journal of Conflict Resolution. 49(4), pp. 625-633. 
Collier, Paul y Nicholas Sambanis (2005). Understanding Civil War. Evidence and Analysis, Vol. 1: Africa. Washington D.C.: The World Bank.

Dealbook (2008). «U.S. Unveils Takeover of Fannie and Freddie». The New York Times. September 7. Consulta: 12 de octubre de 2016.

http://dealbook.nytimes.com/2008/09/07/us-unveils-takeover-of-fannie-and-freddie

Dunning, Thad (2008). Crude Democracy: Natural Resource Wealth and Political Regimes, Cambridge Studies in Comparative Politics. Nueva York: Cambridge University Press.

EIU (2010). Democracy in retreat: A report from the Economist Intelligence Unit. Edición online: The Economist. Consulta: 12 de octubre de 2016.

http://graphics.eiu.com/PDF/Democracy_Index_2010_web.pdf

El País (2011). «La Producción de Petróleo de Libia cae en Picada». El País. Viernes, 25 de febrero, p. 8.

Fearon, James (2005). «Primary Commodity Exports and Civil War». Journal of Conflict Resolution. 49 (4), pp. 483-507.

Gelb, Alan y World Bank (1988). Oil windfalls: blessing or curse? New York/ Oxford: Oxford University Press.

Guriev, Sergei, Antón Kolotilin y Konstantin Sonin (2008). «Determinants of Expropriation in the Oil Sector: A Theory and Evidence from Panel Data». En Discussion Paper 6755. Londres: Centre for Economic Policy Research.

Haber, Stephen y Victor Menaldo (2011). «Do Natural Resources Fuel Authoritarianism? A Reappraisal of the Resource Curse». American Political Science Review. Vol. 105, No. 1, pp. $1-26$.

Humpreys, Macartan y Habaye Mohamed (2005). «Senegal and Mali». En Collier, Paul y Nicholas Sambanis. Understanding Civil War. Evidence and Analysis, Vol. 1: Africa. Washington D.C.: The World Bank.

Isham, Jonathan, Michael Woolcock, Lant Pritchett \& Gwen Busby (2002). "The Varieties of Rentier Experience: How Natural Resource Export Structures Affect the Political Economy of Economic Growth». Working paper \# 12.

http://www.middlebury.edu/media/view/206921/original/IshamPaper.pdf

Kahhat, Farid (2007). «¿Qué Explica el Desempeńo Electoral de la Izquierda en América Latina?». Coyuntura. Año 3, número 13.

Karl, Terry (1997). The Paradox of Plenty: Oil Booms and Petro-States. Berkeley: University of California Press.

Levi, Margaret (1988). Of Rule and Revenue. Berkeley/London: University of California Press.

Mahon, James (2003). The Fiscal Contract, International Capital Flows, and the Quest for the Liberal State. Documento de trabajo. México D.F.: CIDE.

Mann, Michael (1988). States, War and Capitalism. Londres: Basil Blackwell.

Mehlum, H., K. Moene, y R. Torvik (2006). «Institutions and the Resource Curse». Economic Journal. Nro. 116, pp. 1-20.

Moreno-Bird, Juan Carlos e Iván Paunovic (2006). «La Política Económica de la Nueva Izquierda en América Latina: ¿Vino Nuevo en Odres Viejos?». Nexos. Noviembre, pp. 31-37.

Ross, Michael (2003). "The Natural Resource Curse: How Wealth Can Make You Poor». En Bannon, I. y P. Collier (eds.). An Economic History of Twentieth-Century Latin America. The Export Age. The Latin American Economies in the Late Nineteenth and Early Twentieth Centuries. Volume 1. Oxford: Palgrave.

Ross, Michael (2004). «What Do We Know about Natural Resources and Civil War?». Journal of Peace Research. 41(3), pp. 243-252. 
Rueschemeyer, Dietrich, Evelyne Stephens y John Stephens (1992). Capitalist Development and Democracy. Chicago: University of Chicago Press.

Sachs, Jeffrey y Andrew Wagner (1997). Natural Resource Abundance and Economic Growth. Cambridge: Center for International Development and Harvard Institute for International Development, Harvard University.

Sachs, Jeffrey y Andrew Wagner (2001). "The Curse of Natural Resources». European Economic Review. 45(4-6), pp. 827-838.

Sinnott, Emily, John Nash y Augusto de la Torre (2010). Natural Resources in Latin America and the Caribbean, Beyond Booms and Busts? Washington D.C.: The World Bank.

Sala I Martin, Xavier y Arvind SUBRAMAIN (2003). «Addressing the Natural Resource Curse: An Illustration from Nigeria». NBER Working Paper. No. 9804.

The Economist (2008). «Order in the Jungle. Economics and the rule of law». Web, may 13.

http://www.economist.com/node/10849115

Thorp, Rosemary y Bertram Thorp (1978). Peru, 1890-1977: Growth and Policy in an Open Economy. Londres: MacMillan.

Thorp, Rosemary (1991). Economic Management and Economic Development in Peru and Colombia. Pittsburgh, Pa.: University of Pittsburgh Press.

Walt, Stephen (2011). «Social Science and the Libyan Adventure». Foreign Policy. March 24.

http://walt.foreignpolicy.com/posts/2011/03/24/social_science_and_the_libyan_adventure

Weinthal, Erika y Pauline Jones Luong (2006). «Combating the Resource Curse: An Alternative Solution to Managing Mineral Wealth». Perspectives on Politics. Vol. 4, No. 1, pp. 35-53.

Zinn, Annalisa (2005). «Theory versus Reality: Civil War Onset and Avoidance in Nigeria in 1960». En Collier, Paul y Nicholas Sambanis. Understanding Civil War. Evidence and Analysis, Vol. 1: Africa. Washington D.C.: The World Bank.

Zurcher, Christoph, Pavel BAEV y Jan Koehler (2005). «Civil Wars in the Caucasus». En Collier, Paul y Nicholas Sambanis. Understanding Civil War. Evidence and Analysis, Vol. 2: Europe, Central Asia and Other Regions. Washington D.C.: The World Bank. 